\title{
Strates
}

STRATES Matériaux pour la recherche en sciences sociales

Hors-série | 2002

Parcours dans la recherche urbaine, Michel Rochefort, un géographe engagé

\section{Mai 68 : ruptures à l'institut de Géographie}

\section{Nicole Mathieu et Stéphane Cartier}

\section{(2) OpenEdition}

Journals

Édition électronique

URL : http://journals.openedition.org/strates/572

DOI : $10.4000 /$ strates. 572

ISSN : 1777-5442

Éditeur

Laboratoire Ladyss

Édition imprimée

Date de publication : 1 janvier 2002

ISSN : 0768-8067

Référence électronique

Nicole Mathieu et Stéphane Cartier, « Mai 68 : ruptures à l'institut de Géographie », Strates [En ligne], Hors-série | 2002, mis en ligne le 18 mai 2005, consulté le 08 septembre 2020. URL : http://

journals.openedition.org/strates/572 ; DOI : https://doi.org/10.4000/strates.572

Ce document a été généré automatiquement le 8 septembre 2020

Tous droits réservés 


\title{
Mai 68 : ruptures à l'institut de Géographie
}

\author{
Nicole Mathieu et Stéphane Cartier
}

1 Nicole Mathieu: J'ai choisi de m'attacher à un aspect de toi pour lequel j'ai énormément de respect, ton sens politique, ta capacité à comprendre une situation, des relations entre des groupes et des gens, ta capacité à percevoir des rapports de force et à dénouer des contradictions. Je me souviens comment, président du $\mathrm{CSU}^{1}$ alors que celui-ci était presque entièrement passé à droite, tu conservais une ligne droite, précise, conforme à ton appartenance à la gauche, tout en parvenant à élaborer, à proposer des solutions et à les faire adopter. Cependant, plutôt que de m'en tenir au CSU, je préférerais que nous revenions sur ce qui s'est passé en 68 à l'Institut de géographie. Car à cette époque tu es apparu comme quelqu'un qui, conciliateur, a su mettre en perspective des gens qui n'avaient absolument pas les mêmes positions.

2 Commençons par le commencement. Comment ai-je vécu les débuts de 68 ? Pourquoi et comment je m'y suis intéressé au point d'y participer dans la limite de mes capacités? En quoi j'ai pu y avoir un rôle de conciliateur apparent? Ce qui m'amènera à parler d'un «travers » personnel que je connais bien: la séparation, voire la contradiction, entre des idées sociales solidement ancrées, et qui sont restées permanentes, et mes relations interpersonnelles avec ceux qui ne partagent pas mes idées. Pourquoi je suis à la fois empreint d'idées relativement révolutionnaires et, de par mon comportement, relativement tolérant, faisant même preuve d'hypocrisie, de tricherie dans mes relations interpersonnelles avec des gens que pourtant j'enverrais bien au goulag. Politiquement, s'entend !

31968 ! Autant l'interprétation - j'y ai souvent pensé ensuite, j'en ai reparlé avec quantité de gens - demeure présente, autant les faits, les souvenirs précis, risquent parfois d'être déformés au service d'une justification... Quelle que soit la lucidité que j'essaie d'avoir sur moi-même, il y a forcément un système de justification. On ne porte pas un regard complètement objectif sur soi-même.

4 Commençons par cette vision personnelle, ensuite nous pourrons confronter les faits que nous avons vécus et la façon de les interpréter. 
Ce qui me vient à l'esprit immédiatement, et qui manifeste cet esprit conciliateur, c'est l'organisation des examens. En juin 68, il fallait faire en sorte que les étudiants ne pâtissent pas trop des événements. Jean Dresch ${ }^{2}$ m'a confié cette tâche. J'ai essayé de trouver des collègues qui acceptaient de concilier les exigences, malgré tout très fortes de ces chers étudiants, et un cadre légal qui permette que les examens ne soient pas remis en cause. Sur ce point, mes souvenirs sont précis et je reconnais que j'ai eu des idées parce qu'ils m'en ont fait baver! Quand au reste, il demeure malgré tout personnel.

6 Comment me suis-je intéressé à 68 ? Quels facteurs m'ont poussé à « adhérer » (ou à me laisser entraîner) et à agir, étant donné que j'étais à la fois celui contre qui 68 s'élevait et celui qui s'intéressait quand même à ce mouvement?

7 Revenons en arrière. En octobre 1964 je suis nommé à ce qu'on appelait pompeusement la Sorbonne. J'en suis très content ; voire très fier d'évoluer parmi de vieux mandarins dont, c'est vrai, j'avais horreur mais dont j'étais tenté d'adopter les comportements. C'était confortable de bénéficier de ce respect - certes superficiel, mais quand même... de cette relation étudiant/prof et assistant/prof à la Sorbonne. D'autant que c'était parfois d'anciens copains d'études qui se trouvaient être les assistants de ce «jeune » qui arrivait de Strasbourg - j'étais prof à Strasbourg avant - comme professeur à la Sorbonne : tout ça m'a monté à la tête, reconnaissons-le.

8 Pourtant la contradiction entre des irritations, des idées et, disons le, des positions anti-mandarinales - parce que je les ai malgré tout toujours détestés - et une certaine propension à trouver la position confortable et du coup à faire le joli coeur auprès des autres mandarins s'accentue. En ce sens ma relation avec Jacqueline Beaujeu est exemplaire.

9 C'est vrai, on ne peut pas ne pas évoquer cette époque sans rappeler ce que l'on appelait le mandarinat et cette tension d'alors entre l'attrait du mandarin et son rejet. Selon moi, la personnalité de Jacqueline Beaujeu-Garnier est une des pièces fondamentales du tableau de l'Institut.

10 Et je suis relativement réservé sur le rôle objectif qu'elle a joué. Pourtant je n'ai jamais osé le dire, et j'ai toujours été son ami.

11 Parce que tu es capable de vivre très profondément la position de l'autre, même si elle est en contradiction avec la tienne, sans que ça se manifeste violemment.

12 Je pense avoir la capacité de jouer le jeu de l'autre. Je vois bien son système, ses valeurs, j'entre dans son jeu, et jamais je ne me fâche parce que je n'ai pas le moindre souci de le convaincre.

1368 arrive, je ne m'y attendais vraiment pas. Je jouais gentiment mon petit jeu de bon mandarin quoiqu'ayant une propension à faire des choses qui sortaient du mandarinat. C'était les débuts de cette fameuse équipe de géographie humaine dans le $\mathrm{CRDCG}^{3} \mathrm{de}$ Jean Dresch où j'avais déjà rencontré des jeunes et des thèmes nouveaux. Collaborer avec les services d'aménagement m'intéressait. Il y a avait des ouvertures et, je crois, une participation des jeunes dans les équipes. Mais en même temps je ne remettais en cause ni la structure de l'université, ni les fondements mêmes de ma position dans l'université.

14 Pour moi, 68 a représenté deux choses, qui m’ont entrainé au départ pour se concrétiser ensuite. La première c'est qu'il y avait des jeunes qui osaient faire ce que je n'avais jamais osé, gueuler à la face des gens qu'ils étaient des « cons ", qu'il fallait tout 
renverser. Être un révolutionnaire, ce n'était pas simplement un porteur d'idées révolutionnaires issues de ses frustrations ou de je ne sais quoi - ce qui était mon cas mais quelqu'un qui osait s'affirmer comme tel. D'un seul coup, j'ai vu des gens qui avaient le courage, que je n'avais pas, de passer des idées à l'acte révolutionnaire. Ça m'a plu. Même si j'avais la trouille à chaque défilé et qu'une fois je me suis caché dans une église, avenue Parmentier, parce que les flics commençaient à tabasser (rire). J'ai surtout suivi et si je me suis laissé aller à participer un peu, j'étais surtout éminemment intéressé, enthousiasmé, par ces gars qui étaient capables de faire des barricades, d'affirmer leurs différences, de ne plus rien respecter - car, au fond, j'en avais assez de ce respect hypocrite des positions et de la société en place, des bourgeois et de tout ce que je détestais depuis toujours et avec lesquels j'étais, jeune professeur à la Sorbonne, obligé de composer. On ne fait pas sa carrière sans composer, surtout à cette époque. D'un seul coup, apparaissent enfin des hommes... J'avais envie d'être de leur côté, même si je n'étais pas très actif, de me libérer du poids des hypocrisies, des couleuvres que j'ai dû avaler. On en revient à mon élection à la Sorbonne : j'ai quand même eu l'unanimité des onze géographes ${ }^{4}$ de la Sorbonne au moment de la préélection faite par les spécialistes!

15 La seconde chose, c'est qu'en même temps, il y avait une ambiance de fête, ce qui n'est pas, comme vous le savez, pour me déplaire. Comme cela transparaît dans mon comportement et mes discours, j'adore la fête dans tous ses aspects. Et 1968, ça été la fête. Très vite, étant donné que j'adore également les jeunes femmes (rires), ce professeur de quarante ans, auréolé du prestige du mandarin passé du côté de 68 , se retrouvant entouré de jeunes femmes d'une vingtaine d'années, s'est trouvé à l'aise...

La fête dans tous ses états. Gros rouge et saucissonade à l'Institut. Adorant la fête, j'étais prêt à la faire. Sauf avec Jean Dresch, qui avait adhéré à 68 pour d'autres raisons certainement plus nobles que les miennes, mais qui en même temps l'ont un peu paralysé, vis-à-vis des collègues, je jouais un jeu: «Voyez vous, moi, j'essaie de participer, mais c'est pour comprendre et sauver les meubles »... Tu avais pris cette position?

18 Oui, quand ils m'ont annoncé ${ }^{5}$ que c'était ainsi qu'ils me comprenaient, j'ai confirmé que c'était ainsi qu'il fallait me comprendre. J'étais celui qui était plus capable qu'eux d'entendre le mouvement et par conséquent de participer pour sauver les vraies valeurs.

19 En 68, j'ai 32 ans et je n'en suis encore qu'au tout début de ma carrière, petite CR2 rentrée depuis moins d'un an au Cnrs, je suis loin d'avoir ta position au sein de l'Institut et sur le mandarinat. Pourtant n'étant plus étudiante, je me sens déjà de l'autre bord. Or les deux points que tu avances sont effectivement et incontestablement également très forts pour moi. J'étais fascinée de voir des gens qui avaient le courage de parler et de dire des choses que je n'aurais pas osé dire. En plus, ce sont des femmes, et certaines m'éblouissent, Hélène Lamicq, Bazire etc., ce groupe de jeunes filles à la parole si libre, qui pesait si naturellement sur les assemblées de l'amphi De Martonne. L'autre aspect, c'est la fête. Je reconnais aussi le goût de ces jours passés dans l'Institut devenue notre maison animée et joyeuse. Mais, je vois aussi un troisième aspect que tu n'évoques pas: dès les premiers jours de Mai 68, j'ai eu le sentiment d'une marge d'action, qu'il était possible de refaire la structure, de refaire le monde, avec mes collègues du Cnrs, les ingénieurs comme les chercheurs, avec les enseignants, assistants et maîtres-assistants, avec les autres Comités de grève et groupes universitaires à la Sorbonne ou à Jussieu... 

ces positions et de ces valeurs face à une société qui, dans son immense majorité, n'avait aucune envie de changer, de l'inanité de la révolution du petit nombre... Mais en même temps je ne serai plus jamais le même du point de vue de cette capacité de double jeu. Je serai plus ferme sur un certain nombre de valeurs et de positions, moins fuyant. Certes j'en ai encore trompé un certain nombre. Du coup, je me suis fait beaucoup d'ennemis. Avant, je n'avais aucun ennemi, comme le prouve mon élection à l'unanimité à la Sorbonne ! Après 68 , tout a été plus net mais dans une position qui était moins extrême. Je suis arrivé à un compromis avec moi-même vis-à-vis de ce qu'on pouvait gagner dans la société, je suis redevenu participationniste, c'est pourquoi j'ai accepté de présider le CSU en 1982 (le CNU actuel) mais avec des idées qui pouvaient trouver sinon une majorité, du moins un appui suffisant pour que je puisse les défendre sans être obligé de trop tricher avec les autres. La preuve, c'est qu'ils ont quand même mené une sacrée campagne contre moi. 
iciper, c'était pérenniser les institutions que l'on condamnait. Plus on participe, plus on repousse l'échéance qui verra tout s'effondrer pour une société nouvelle. Ce qui était également vrai en ce qui concerne ma participation à l'aménagement du territoire où je me suis aperçu, et j'ai tenu ce discours au Brésil pendant des années, que participer permet, certes, de gagner sa vie, mais participer c'est également apaiser un certain nombre de contradictions de la société, c'est par conséquent reculer l'échéance où ces contradictions la feront éclater. Il est clair qu'il s'agissait pour moi de retrouver un espoir après la faillite de mon premier espoir dans ce domaine - il ne faut pas oublier que j'ai été communiste en 1944-45 et jusqu'en 56. À cette époque, il n'était pas question de réformer la société. Nous étions pris par l'idée de faire du passé table rase. Même si nous évitions, au moment de la guerre froide, de nous retrouver en prison, nous étions révolutionnaires et staliniens. On admirait Staline. Pour le peuple, magnifié, il fallait apporter une nouvelle société communiste et donc être radical, briser pour construire. Ce rêve s'effondre, pour moi, en 56 . Quand nous nous sommes aperçus que le peuple voulait autre chose et qu'on lui envoyait des tanks, ce fut la faillite totale de l'engagement et du bouillonnement de mon enfance et de mon adolescence, de tout ce qui explique mon horreur des bourgeois et surtout des collabos. Ce sont des haines profondes. Celles-ci semblaient pouvoir se concrétiser, s'engager, dans l'idée qu'on allait tout changer. C'est l'engagement communiste de 44-45. En 56, tout s'effondre et même si cette force épicurienne, que je tiens sans doute de mes ancêtres bourguignons, m'a permis de me réconcilier avec moi-même en faisant la fête, je suis devenu infiniment plus cynique, j'avais perdu mes bases. De 56 on tombe facilement à 64 , où ce cynisme, compensé par la fête, se réalise dans ma carrière. 68 , c'est aussi la découverte d'une dimension autre de moi-même.

Les événements de 68 sont donc, pour toi, une façon de te réconcilier avec des valeurs propres et avec le politique dans ta personnalité. Mais au moment même, en juin, juillet et septembre, quelles sont tes pratiques? J'ai, quant à moi, des souvenirs extrêmement précis de 68 sur le plan pratique. Or, toi, tu ne gardes, en tant qu'acteur, de souvenir de fait précis que les examens...

Acteur dans le compromis. Sinon j'étais suiveur, j'écoutais, passionné par ce qui se disait, et je ne prenais que très rarement la parole. Quand cela m'arrivait, c'était pour dire des bêtises. Je n'avais pas grand chose à dire. Je participais en tant que présence à ce qui se faisait, comme l'occupation de l'Institut. J'ai dormi des jours et des jours dans un lit pliant dans le bureau de Pierre George (rire). J'ai participé à un certain nombre de manifestations.

31 Tu ne vois dans cette période-là que les étudiants, les amphis, les jeunes assistants... Est-ce que tu vois ce qui se passait au CRDCG?

Pas du tout.

Non! C'est extraordinaire. Tu as oublié que nous avons fait une nouvelle constitution de l'Institut?

Non, je ne me souviens plus. Je n'y ai pas participé, je ne crois pas. Ou alors j'ai écouté. La seule chose dont je me souvienne pour laquelle j'ai été réellement constructeur, ce 
sont les examens, dont l'enjeu était important puisqu'on savait déjà que 68 c'était foutu et qu'il fallait sauver les meubles. C'est vrai qu'à ce moment-là je me suis mobilisé.

Ce que ça révèle, c'est que tu étais anti-mandarinal et en cela parfaitement inscrit dans le mouvement de 68, tu étais sensible à tout ce qui se passait en grand collectif, mais tu n'as pas perçu ce qui, en quelque sorte, était le niveau intermédiaire, l'organisation interne de l'Institut avec essentiellement des gens qui en étaient au tout début de carrière, ou alors les ITA ${ }^{6}$.

Je me souviens maintenant qu'on avait fait une réforme du fonctionnement de l'enseignement à l'Institut où j'avais été nommé vice-directeur, Dresch étant directeur. C'est cela.

Et c'est ça qui m'a donné la possibilité d'organiser les examens.

Nous avions organisé des élections à l'Institut.

Oui, j'ai été candidat «muet » et vous m'avez élu. Mais, bien que j'ai dû y participer, je ne me souviens pas des phases de l'organisation.

41 Donc cela ne t'intéressait pas?

Ou je ne me sentais pas à l'aise, je ne sais. Je ne peux pas te répondre, je ne m'en souviens pas.

43 Il y a eu quelque chose de très réformiste dans le mouvement de 68. L'aspect révolutionnaire était incontestablement accompagné d'un réformisme "du local ", un réformisme des rapports immédiats, un réformisme pour lequel changer le monde c'était changer les rapports avec les étudiants, les enseignants, etc. Et toi, tu étais celui qui nous permettait de le faire. En réalité, nous étions horriblement divisés, trotskistes, maos... Il y avait énormément de conflits à l'intérieur...

44 Je ne les voyais pas. J'étais davantage intéressé par ce que pensait Hélène Lamicq et à me laisser aller, sans trop prendre conscience des conséquences, à adhérer à tout ce qu'elle proposait. On pourrait dire que c'est une période de ma vie où je ne me suis pas penché sur les tenants et les aboutissants, contrairement à ce que j'ai toujours fait, et où je me suis laissé emporter dans un courant passionnel vis-à-vis d'idées qui me séduisaient et d'une femme qui les exprimait et les incarnait. Je ne me pensais pas comme un acteur. Au fond, je ne sais pas moi-même ce qui a fait que j'ai pu mettre mon intérêt personnel et ma position de prof entre parenthèses. Je ne me posais pas la question des conséquences. Ce n'est qu'après, bien content malgré tout de me retrouver prof comme avant (rire), que j'ai retrouvé l'idée de l'action et du fait que je pouvais être utile. J'étais séduit par des idées, par une personne.

Je n'ai pas été un acteur de 68. Parce que vous m'y avez poussé, je suis devenu vicedirecteur de cet Institut réformé. Quand à l'organisation elle-même, je n'ai pas de souvenir de ces divisions... Cela devait se passer sans moi. Cela se disait dans les amphis.

47 Je n'y étais pas toujours et souvent j'y faisais des bateaux en papier, ce qui est tout de même un symbole! Avec une feuille 21-27 je faisais jusqu'à six pliages de bateau tout en vous écoutant, ce qui témoigne d'un certain détachement. Était-ce qu'au fond de moi je savais qu'il fallait les laisser gueuler et attendre pour retrouver sa place de mandarin? Étais-je passionné sans pour autant me sentir capable d'être acteur? J'ai toujours été impressionné par les révolutionnaires qui avaient des structures. J’ai été communiste, mais du marxisme je n'avais lu que Les problèmes économiques et sociaux de Staline. J'ai très peu de culture marxiste. Se dire marxiste allait de soi quand on était communiste. 
Il se trouve qu'une certaine approche de la géographie sociale aboutit à retrouver des structures et des mécanismes dialectiques qui peuvent rappeler le marxisme, mais on collait l'étiquette marxiste à tout va. J'ai toujours eu un certain complexe vis-à-vis des vrais marxistes comme Dresch ou George. Je n'ai jamais lu Le capital. Qu'on l'ait lu m'impressionnait. J'avais, en 68 , un peu peur de ceux qui semblaient posséder une culture et une structure idéologique. Mes pulsions m'entraînaient dans cette direction, je trouvais vraiment sympa de foutre en l'air cette société que je détestais, mais je n'avais pas de bases idéologiques. Poussé, j'ai agi, mais je ne crois pas avoir été acteur de l'application d'une certaine idéologie à la réforme de la société.

Stéphane Cartier: Quels étaient ces meubles qu'il fallait sauver au point de s'engager dans l'organisation des examens?

49 Ce n'est pas moi, c'est le groupe Sautter, Beaujeu, etc. qui voulait les sauver. On sait que Pierre George s'était réfugié à Chatenay-Malabry et n'a jamais voulu venir. Il ne faut pas l'oublier.

$50 \quad$ N. M. : Les géographes communistes se sont divisés en 68. Je me souviens que Guglielmo a déchiré sa carte sur les marches de la Sorbonne.

Gugli est resté communiste jusqu'en 68. Il était extrêmement engagé et passionné par toutes ces idées qui s'exprimaient et cette jeunesse qui nous enthousiasmait... Toujours exalté quand il s'agit des mouvements du peuple (rire), considérant que le parti communiste trahissait cet enthousiasme, il a fait sa crise, comme moi lorsqu'en 56 j'ai pensé que les tanks trahissaient l'enthousiasme d'un peuple. Les communistes trouvaient qu'on était des irresponsables, de dangereux révolutionnaires et je faisais partie du lot.

Il y a eu, dans le petit monde de l'Institut, cinq tendances :

53 - ceux qui refusaient tout parce qu'ils étaient politiquement de droite ou d'extrême droite et tenaient à sauvegarder l'archaïsme de la Sorbonne traditionnelle. Ce sont eux qui iront à Paris IV au moment de la séparation ;

54 - il y a ensuite ceux qui, politiquement, étaient centristes, et qui, en fait, voulaient, consciemment ou inconsciemment, sauver leurs privilèges de mandarin, ou éventuellement, préserver quelques valeurs, menacées par 68, auxquels ils croyaient. Selon eux, laisser les étudiants prendre le pouvoir, c'était compromettre le niveau de l'université. Ce qui suppose qu'il y en ait qui savent, et enseignent, et d'autres qui sont là pour apprendre, qu'aucun dialogue n'est possible puisque ceux qui doivent apprendre, il leur faut écouter, et que ceux qui ont la capacité de transmettre doivent pouvoir parler librement. Aucun compromis n'était donc acceptable sur la question du niveau et donc des examens. Aucun compromis également dans la relation enseignant/ enseigné puisque tous deux n'ont pas le même niveau, intellectuel, s'entend. De là il découle qu'il faut sauver les structures, peut-être doivent-elles être améliorées, mais il faut que les profs restent des profs et les assistants, des assistants. "Nous, nous avons fait nos preuves, nous avons un doctorat d'État, il ne faut tout de même pas nous mettre sur un même niveau... ». Nous, notre idée, c'était le cadre unique, un cadre unique qui organise l'institution avec une participation active des étudiants. C'était précisément ce qu'ils voulaient éviter. Pas question d'une participation étudiante. Pas question d'un cadre unique parce qu'il y a dans les niveaux une hiérarchie entre ceux qui ont fait leur preuve et ceux qui n'ont encore rien prouvé. Ce qu'ils voulaient sauver, c'était leur pouvoir. Il n'y avait dans ces deux groupes que des profs. Les assistants 
étaient perçus par les mandarins comme des gens qui, profitant de 68 , cherchaient à prendre plus de pouvoir. Et pour ceux qui acceptaient des réformes, c'est qu'ils comprenaient qu'il fallait lâcher du lest ;

- le troisième groupe rassemble des gens, qui sont plutôt de gauche ou tout à fait de gauche comme Pierre George ${ }^{7}$ ou Pierre Monbeig $^{8}$, mais qui rejetaient Mai 68, parce qu'ils se sentaient atteints dans leur honneur ou dans leurs certitudes ; - le quatrième groupe, les communistes. Je suis persuadé qu'ils avaient des réunions fractionnelles entre communistes. Mais ils étaient en même temps avec nous, avec les enseignants qui participaient (cinquième groupe) plus ou moins.

57 Je suis le seul mandarin, le seul prof qui ait participé. J'adhérais complètement sans aucune réticence. J'ai vécu 68 sans avoir l'impression d'avoir quoi que ce soit à sauver. Je m'étais complètement engagé dans quelque chose de nouveau. Si je demeure l'unique professeur, en revanche, pratiquement tous les assistants, les chargés de recherches, etc. se retrouvent dans la catégorie de ceux qui adhèrent. Ils y sont presque tous. Avec leurs différences...

59 Dissensions internes que je perçois mal. Je suis, naïf et utopique, entièrement dans le mouvement.

60 Ce n'était pas des dissensions mais des positions différentes que l'on retrouvera par la suite. Les ITA et les chercheurs étaient très fortement pour toi. Dans le mouvement de 68, il y a, selon moi, la question du collectif et cette recherche d'une position collective. Tu étais celui qui nous permettait de faire passer cette idée. Ce n'était pas les jeunes, mais nous, la génération intermédiaire qui la défendions. Nos références, c'était 56 certes, mais surtout l'Algérie. C'était l'occasion de reconstruire quelque chose tout en composant avec des contradictions fortes.

61 Revenons aux examens. C'était à la fin juin.

62 On arrive à un moment où les gens lucides savent que c'est fichu, à partir de la mi-juin, et où se pose le problème des examens. Commençons par les acteurs principaux : il $\mathrm{y} a$ une demande très forte des étudiants qui ne veulent pas perdre leur année. Demande très ambiguë puisqu'elle va se heurter à des conceptions différentes en ce qui concerne la notion d'examen. Les radicaux refusent la notion d'examen.

Il y avait même des défilés où l'on demandait la suppression de l'agrégation.

64 Il y avait un mouvement général de contestation des examens, pourtant ils voulaient qu'on leur donne leur année. Il y avait également ceux qui étaient prêts à passer des examens traditionnels à condition qu'on allège les programmes puisque les cours n'avaient pas eu lieu. Parce qu'il y a aussi des étudiants réactionnaires. Ils se manifestent à ce moment là. Et puis, il y a ceux qui veulent de vrais examens mais repensés, réorganisés. Avec une forte demande de participation de délégués étudiants aux jurys. C'était la grande idée. Ils comptaient sur moi. Très vite je leur ai dit qu'il était impossible d'obtenir une participation étudiante. Dresch était très ferme également. On a fini par trouver un accord selon lequel ils renonçaient à la participation, mais à condition qu'il y ait une attention particulière, une autre façon d'interroger, qu'on essaie de faire plutôt des oraux que des écrits et qu'il y ait une adaptation partielle de la structure de l'examen. Il me semble, je n'en suis pas sûr, que les étudiants demandaient un rôle plus important des assistants. Avant 68, c'était le prof qui interrogeait, en présence d'un assistant, mais celui-ci n'avait pas son mot à dire au moment de la note. Il n'était là que pour la forme. 

et étudiants, à la suite de 68. Les étudiants renoncent à la participation, c'est-à-dire au contrôle que nous allons demander au Cnrs. Partout on cherche à obtenir que ceux qui sont concernés entrent dans les structures, ne serait-ce qu'à titre d'observateur. les étudiants sont présents, et le début du contrôle continu des connaissances qui permet de tenir compte du travail de l'année dans la notation et d'éviter que les examens soient un couperet final.

En juin, dans le groupe le moins traditionnel, celui d'Hélène...

Hélène se rallie à ma position acceptant que l'on ne puisse être à la fois juge et partie. Il y aura des étudiants examinés et des examinateurs, mais il faut que la façon d'examiner soit réorganisée. Ils lancent déjà l'idée qu'il faudra tenir compte d'un système de jugement permanent. Ce n'est encore qu'une idée. Pour ce qui est des examens de juin 68 , il a fallu trouver des jurys qui acceptent une participation plus active des assistants et acceptent de tenir compte des conditions exceptionnelles de 68. Définir des programmes allégés qui ne concernent que les deux premiers trimestres. On crée alors une espèce de commission à laquelle participent les étudiants et un certain nombre d'assistants. Ce qui bouleversait profondément les professeurs parce que les examens, c'était leur pouvoir, ce pouvoir discrétionnaire qui permettait de décider si un étudiant méritait ou non son examen, ce pouvoir de juger.

Les étudiants eux-mêmes étaient d'accord, on ne pouvait organiser les examens sans un certain nombre de profs, d'autant que les assistants ne pensaient pas pouvoir constituer de jurys s'il n'y avait pas au moins un prof.

C'est vrai que j'ai joué le jeu, affirmant que pour sauver les meubles, il fallait lâcher du lest, organiser des examens honorables et même si c'était contraire à leur principe, un certain nombre de professeurs ont accepté. Je ne suis pas allé plus loin en direction de certains mandarins physiciens qui n'étaient plus revenus à l'Institut depuis le début mai. Nous avons fait passer les examens sans eux avec suffisamment de jurys cependant pour que tout se déroule à peu près honnêtement. Nous sommes restés dans des normes de respect des critères habituels. Certains étudiants ont été collés, d'autres ont reçu des mentions méritées. Tout s'est à peu près bien passé, sur des bases de programme allégé, mais avec des jurys d'enseignants jugeant des étudiants.

Tu as donc eu un rôle essentiel...

Oui, sûrement.

... de médiateur entre les positions les plus extrémistes, certains étudiants demandaient qu'on accorde l'année sans même faire passer d'examen...

C'était la position majoritaire des étudiants engagés au début. Ils ont vu que ce n'était pas possible...

C'est toi qui faisais face?

Oui, parce que je savais que si on leur donnait l'examen, il serait cassé. On ne pouvait déjà plus croire à 68 . Je voyais bien que les mandarins étaient tout près à reprendre le pouvoir. Je n'imaginais même pas qu'on aurait le compromis d'Edgar Faure l'année suivante. Il me semblait que les mandarins allaient revenir en force, relever la tête et imposer de nouveau la structure antérieure. C'est vrai que le compromis d'Edgar Faure, compromis des politiques, a obligé la grande majorité des universitaires à mettre de 
l'eau dans leur vin et à accepter d'imaginer de nouvelles structures pour l'université. Tous ceux qui étaient contre toute nouveauté se sont retrouvés à Paris IV s'emparant $\mathrm{du}$ mot Sorbonne pour signifier qu'ils demeuraient dans la tradition. Plusieurs professeurs de l'Institut ont opté pour Paris IV (Birot, Pédelaborde, Guilcher...) accompagnés par quelques rares assistants.

$T u$ prends la suite d'une des parties du CRDCG.

78 Le CRDCG explose à la rentrée de 68, dissociant un laboratoire de géographie physique, un laboratoire de géographie humaine et un laboratoire de cartographie. On me propose d'être directeur du laboratoire de géographie humaine, et j'accepte alors que j'avais refusé d'être directeur de l'Institut. Il s'agissait cette fois de la recherche et j'avais l'impression que celle-ci avait déjà assez avancé pour qu'on ne retombe pas dans des structures mandarinales. Il me semblait important de la favoriser parce qu'elle pouvait se faire dans la ligne de 68 .

Pourquoi faisais-tu plus confiance à la recherche?

80 La recherche dépendait des jeunes - j'avais déjà apporté ma contribution - j'y ai cru et je me suis engagé. J'étais plus libre d'un engagement global au sein du Laboratoire, même si mon esprit de conciliation, mon refus de se fâcher, cette façon de ménager la chèvre et le chou et de ne pas prendre de position radicale, ont parfois agacé les plus engagés.

81 Tout choix volontaire étant lourd du regret de toutes les vies possibles qu'il exclut, comme le disait Ferdinand Alquié, mon professeur de philosophie en hypokhâgne, je n'ai jamais réussi à dominer cette nécessité du choix et à accepter d'être privé de ce que je laissais en chemin. Je n'ai donc jamais été complètement engagé. J'ai même toujours cherché à éviter les choix idéologiques qui m'engageaient dans des relations conflictuelles avec les êtres. Je déteste les conflits. Comme je n'ai jamais eu de goût pour l'affrontement interpersonnel, je cherche à biaiser, je joue la carte de la diplomatie, du compromis.

82 S. C. : Fédérateur au niveau de l'Institut et peut-être du Laboratoire parce que pris dans un évitement du conflit...

83 Non, pas fédérateur, conciliateur. Essayant de concilier les inconciliables, j'arrivais à persuader les uns et les autres que j'étais de leur côté. Chacun croyant que j'étais avec lui, j'arrivais à tirer les ficelles.

$84 \quad$ N. M.: Tu savais déployer, au sein du Labo, une argumentation qui reconnaissait, à part entière, la place de chacun, et proposer une solution pratique qui finalement permettait à ces inconciliables de penser que chacun était reconnu. Je me souviens que tu as réussi à obtenir que chaque membre du Labo tienne le calendrier de ses travaux individuels et collectifs qui était présenté et discuté en conseil de Labo.

Oui, ces compromis étaient indispensables.

86 Une capacité à préserver profondément l'absolu (la pureté) de chaque position. Nous avions choisi d'être là, et non à Paris VII ou Paris IV, au côté d'un personnage qui nous paraissait être celui qui serait le plus à même de laisser une respiration à ce mouvement de 68 .

87 Sur le plan du Labo, malgré toutes ses imperfections, nous avons sauvé ce qui pouvait être sauvé au regard du désastre de l'après-68...

88 Aussi bien du point de vue de la qualité de vie que d'objectifs intellectuels comme l'intérêt pour l'industrie, la question de l'analyse des systèmes de pouvoir... Nous avons fait quelque chose de 
notre expérience de 68 transformant à la fois les rapports humains et le rapport au collectif, et, pour certains, leur intérêt pour certaines recherches.

De mon point de vue, celui d'un directeur, cela signifie que j'ai pu utiliser les fonds de l'État pour financer des recherches qui n'étaient plus acceptées par le milieu officiel. J'étais contesté, on m'a taxé de Labo rouge, mais on n'a jamais pu, entre 69 et 84, me déboulonner. Après je suis parti parce que c'était le moment de le faire.

Et, grâce à cela, à l'abri de Michel, se sont constitués des gens et des équipes qui avaient une pureté intellectuelle, une pureté dans la manière de travailler collectivement, et qui sans cela n'auraient jamais pu faire carrière.

91 Ils auraient tout simplement été mis à la porte du Cnrs.

92 Ils ont, bien entendu, eu des misères.

93 S. C. : Qu'entendez-vous par " sauver le principal dans le désastre de l'après-68 »?

94 Le désastre, c'était la reprise en main des structures et des financements de la recherche par les conservateurs qui refusaient tout nouveau thème de recherche et surtout les recherches engagées dans la remise en cause de la société. Sur le plan universitaire l'enjeu est le même, mettre en place des structures qui évitent toute poursuite de la contestation à l'université. Il fallait que l'université soit marginalisée parce qu'indécrottable ou alors remise dans les rails. Avant 81 , à l'université, on a assisté à une reprise du pouvoir par les mandarins, l'apothéose (si j'ose dire) dans la chasse à tout ce qui pouvait être progressiste correspondant à 1978-81. L'absence de promotion faisant partie, bien entendu, des moyens de pression.

Pourtant, au Labo, on ne m'a jamais coupé les financements. J'étais le maître de la distribution des crédits en ce qui concerne les thèmes de recherche. Sans pénaliser les recherches établies, j'ai donné de l'argent à des gens qui, de mon point de vue, faisaient des recherches qui étaient dans la ligne du "progrès » de l'humanité. En ce sens, effectivement, nous avons pu préserver certaines choses du désastre ambiant, de la «restauration ».

96 Les mandarins ont complètement pris le pouvoir en ce qui concerne la gestion de l'université. Nous en avons été victime, c'est vrai. En revanche, parce qu'au Cnrs on avait obtenu la participation des chercheurs dans les structures de gestion, on n'a jamais pu me déboulonner. Nous étions, à chaque fois, plus ou moins mal classés mais malgré tout, renouvelés tous les quatre ans. Lorsqu'on m'a traité de Labo rouge à la commission nationale du Cnrs, il s'en est fallu d'un cheveu, mais ils n'ont pas osé. Sans doute parce que j'avais gardé un certain prestige personnel dans l'université.

$97 \quad$ N. M. : Il faut reconnaître également que, dans la gestion interne du Laboratoire, tu as toujours respecté la diversité des orientations politiques. Philippe Pinchemel, qui n'était pas particulièrement "rouge" représentait son équipe au Conseil de labo et y avait sa place avec moi, une des chercheuses particulièrement rouge du labo, qui faisait partie de son équipe!

Contrairement à la plupart de mes collègues directeurs je n'ai jamais financé mes recherches ni mes déplacements. Je ne me suis jamais accordé de mission sur les fonds du Laboratoire pendant les 17 ans où je l'ai dirigé. J'ai toujours veillé à ce que mes déplacements soient financés par d'autre sources. Il n'y avait donc aucun moyen de m'épingler, ce qui explique peut-être qu'ils n'aient pu me déboulonner. Car c'est toujours très difficile d'accuser quelqu'un de financer des recherches dangereuses... 
99 En 1981, quand Chevènement est devenu ministre de la recherche et a lancé ses Programmes Mobilisateurs dans le cadre du programme mobilisateur 4, j'ai été chargé de créer une commission pour le financement de la Recherche Urbaine dans les PVD. C'est la seule fois de ma vie où j'ai vraiment eu l'initiative totale quant au financement de la recherche sur le fond. Ma grande qualité a été de savoir m'entourer de gens qui allaient plus loin que moi dans ces recherches. J'ai été repris comme président du Comité scientifique du Département Recherche pour le Développement du ministère de la Recherche lorsque Curien est devenu ministre, en 1988. J'ai, petit à petit, essayé de ramener un financement de la recherche urbaine et je réussissais en 1993 lorsque le nouveau ministre a supprimé le Département recherche pour le développement, arguant que « recherche pour le développement » ne voulait rien dire.

S. C. : Rien de personnel dans tout ça?

Oh ! J'avais quand même des pancartes au dos et en particulier, pour eux, la pancarte de 1981, de la commission Recherche urbaine. J'étais celui qui avait été choisi en 1981, et sûrement, le bruit circulait dans les cabinets que j'étais un gauchiste.

102 Sur la question des examens, par exemple, s'agissait-il d'un débat général, partagé par les autres universités, ou d'un contexte particulier à l'Institut de géographie?

Il s'agissait d'un débat à l'intérieur de l'Institut qui a eu une situation particulière. Les débats généraux étaient totalement conflictuels, avec la Sorbonne, occupée essentiellement par les étudiants - peu de professeurs s'y rendaient - puis vidée par les flics et où il n'y avait plus d'étudiants. Tandis que nous, nous avons décidé, avec les gauchistes « raisonnables », d'occuper l'Institut pour empêcher les extrémismes. De ce fait, nous avons fonctionné en vase clos et l'évolution a été moins contrastée ${ }^{10}$. Il n'y a pas eu de saccage, pas d'intervention brutale de la police, un compromis pour les examens etc. On a été vraiment un cas.

\section{ANNEXES}

L'Institut de géographie ressemblait à Jussieu, qui fonctionnait aussi comme une institution, dans l'idée d'une gestion locale et autonome. Je me souviens des derniers jours de juin après les accords de Grenelle. Les ouvriers de Renault ne voulaient pas céder et reprendre le travail. Ils avaient appelé les étudiants et enseignants à manifester leur solidarité en venant sur le site de l'usine de Flins. Le comité de grève de Jussieu décide d'envoyer une délégation pour rencontrer celui de l'Institut de géographie afin de définir le meilleur itinéraire (les petites routes et non l'autoroute qui serait certainement surveillée). La veille de la manif, la délégation arrive dans le hall. Cette nuit, le seul prof qui occupait était Jean Dresch. Il nous fallait son accord pour consulter les cartes de la bibliothèque. Mais Dresch refuse de fournir les cartes à des gauchistes de l'extérieur.

Ceux de Jussieu négocient sur la base d'arguments scientifiques : il faut de l'exactitude et de l'efficacité. Finalement Dresch monte à la bibliothèque et en revient avec les 
cartes qu'on étale dans le hall. Autour de Dresch, tous accroupis, nous avons fait l'itinéraire. Les seules délégations qui ont réussi à contourner les flics et à parvenir à Flins, ce sont celles de Jussieu et de l'Institut, toutes les autres se sont fait arrêter.

Nicole Mathieu

\section{NOTES}

1.CSU (Conseil Supérieur des Universités).

2.Jean Dresch était directeur de l'Institut de géographie en 1968 et du CRDCG (Centre de recherche et de documentation cartographique et géographique). C'était au fond lui le « patron » du 191 rue Saint Jacques avec ses dépendances en préfabriqués.

3.Centre de recherche et de documentation cartographique et géographique.

4.Ces onze professeurs parmi lesquels le chef de file de la gauche était Pierre George et celui de la droite était Jacqueline Beaujeu étaient : Pierre Birot, Jean Delvert, Jean Despois, Jean Dresch, André Guilcher, Fernand Joly, Pierre Monbeig, Pierre Pedelaborde, Aimé Perpillou.

5.Au cours d'une des réunions (rassemblant certains collègues dont Gilles Sautter) qui se tenaient chez Jacqueline Beaujeu. Elle habitait rue Pierre et Marie Curie, à deux pas de l'Institut et des événements qui s'y produisaient.

6.Les Ingénieurs, Techniciens et Administratifs qui pouvaient être dans les labos du Cnrs ou attachés aux professeurs.

7.« On raconte l'anecdote suivante : à l'étranger au début des événements, Pierre George, à son retour, décide de venir dans une assemblée pour exprimer sa désapprobation du mouvement étudiant. L'apprenant, ses 'élèves', Michel Coquery, Raymond Guglielmo, Yves Lacoste, Michel Rochefort décident alors de se rendre chez lui à Chatenay-Malabry un soir après dîner où ils sonnent jusqu'à ce qu'on leur ouvre. Ils lui déclarent que s'il venait demain, ils seraient tous contre lui ; tous ses élèves... Ils ne réussiront pas à vaincre son hostilité et la seule chose qu'ils obtiennent de lui, c'est qu'il ne vienne pas " (Nicole Mathieu).

8.Il était alors directeur scientifique au Cnrs.

9. En onze ans, entre 1970 et 1981, Michel Rochefort n'a eu aucune promotion, la gestion des carrières universitaires étant assurée par le Conseil supérieur des corps universitaires.

10.« Nous occupions aussi la nuit l'Institut pour le protéger de l'extrême-droite, et en particulier d'Occident, qui a tenté de forcer la porte d'entrée (parmi eux se trouvaient Madelin et Longuet). Ils ont été reçus à coup de bouteilles lancées des fenêtres des étages tandis que d'autres bloquaient la porte » (Note de Nicole Mathieu qui était présente ce soir-là). 


\section{AUTEURS}

NICOLE MATHIEU

Ladyss, Cnrs

\section{STÉPHANE CARTIER}

Cnrs 